\title{
Le mura di Leonardo. I rilievi del 1502
}

\author{
Leonardo's Walls. Surveys in 1502
}

\section{Marco Carpiceci ${ }^{a}$, Fabio Colonnese ${ }^{b}$}

Sapienza Università di Roma, Rome, Italy

a marco.carpiceci@uniroma1.it; ${ }^{\mathrm{b}}$ fabio.colonnese@uniroma1.it

\begin{abstract}
In the summer of 1502, Cesare Borgia appointed Leonardo da Vinci for his engineering expertise. His assignment was specific and concerning with military architecture: he was expected to "see, measure and do good estimation". The Codex L, a small notebook conserved in the Library of the Institute of France, show the results of the survey of the city walls of Cesena and Urbino. The technique Leonardo adopted consists in traversing rectilinear stretches, measuring their length by means of an instrument able to count his steps and establishing their orientation by means of a compass. At the end of the path, the data relative to the sides of a closed polygon are obtained, resulting the geometric plan of the walls. This practice is testified by some residual eidotypes provided with quotas and orientations. In some cases, only the lists of distances in numbers are present, but the analysis of the figures makes it possible to reconstruct the surveyed plans, as Nando De Toni pioneered many years ago. This study focuses on the tools and the urban survey technique used by Leonardo. The analysis of some sheets from the Codex L, contextualized with respect to the actual topography of the sites, allows to understand the correct sequence of the operations carried out first in the site and then at the drawing board. By means of specific digital reconstructions, it is therefore possible to study the instrumental and operational limits of this practice and, by comparing it with the current state, to reconstruct the entire defensive structure.
\end{abstract}

Keywords: Leonardo da Vinci, Cesena, Urbino, fortified walls, urban survey.

\section{Introduzione}

Nell'estate del 1502 Leonardo viene assunto da Cesare Borgia. Il suo incarico è specifico e riguarda l'architettura militare (Marani 1984), per la quale dovrà vedere, misurare et bene estima$r e$. Tracce del meticoloso lavoro si trovano in un piccolo taccuino della Biblioteca dell' Istituto di Francia, il Codice L. Si tratta di disegni tracciati in sito che mostrano il lavoro di rilevamento planimetrico; esemplari sono quelli delle cinte murarie di Cesena e di Urbino. Questo studio si concentra sugli strumenti e sulla tecnica di rilevamento urbanistico utilizzata da Leonardo. Essa consiste nel percorrere tratti rettilinei misurandone la lunghezza mediante uno strumento conta passi e di stabilirne l'orientamento mediante una bussola. Alla fine del percorso si ottengono i dati relativi ai lati di un poligono chiuso che è quindi controllabile anche nella sua geometria. Di tale prassi ci rimangono alcuni eidotipi completi di quote e orientamenti. Quando sono presenti solo le liste delle distanze in numeri, l'analisi delle cifre permette di ricostruire le planimetrie rilevate, come indicato da Nando De Toni (1965). L'analisi di alcuni fogli del taccuino L, contestualizzata rispetto alla topografia dei luoghi, consente di risalire alla corretta sequenza delle operazioni compiute prima sul campo e poi al tavolo da disegno. Mediante apposite ricostru- 
zioni digitali, è possibile quindi studiarne i limiti strumentali e operativi e ricostruire l'intera struttura difensiva comparandola con lo stato attuale dei luoghi. Questo esito costituisce la base per poter meglio analizzare e comprendere in futuro altri disegni urbani di Leonardo, come quello relativo al Porto di Cesenatico o la celebre pianta di Imola.

\section{I rilievi del Taccuino $L$}

Nel piccolo taccuino L $(7,5 \times 4 \mathrm{~cm}$, base $\mathrm{x}$ altezza) sono visibili le minute "di campagna" tracciate da Leonardo nel rilevamento delle mura di due città: Cesena e Urbino.
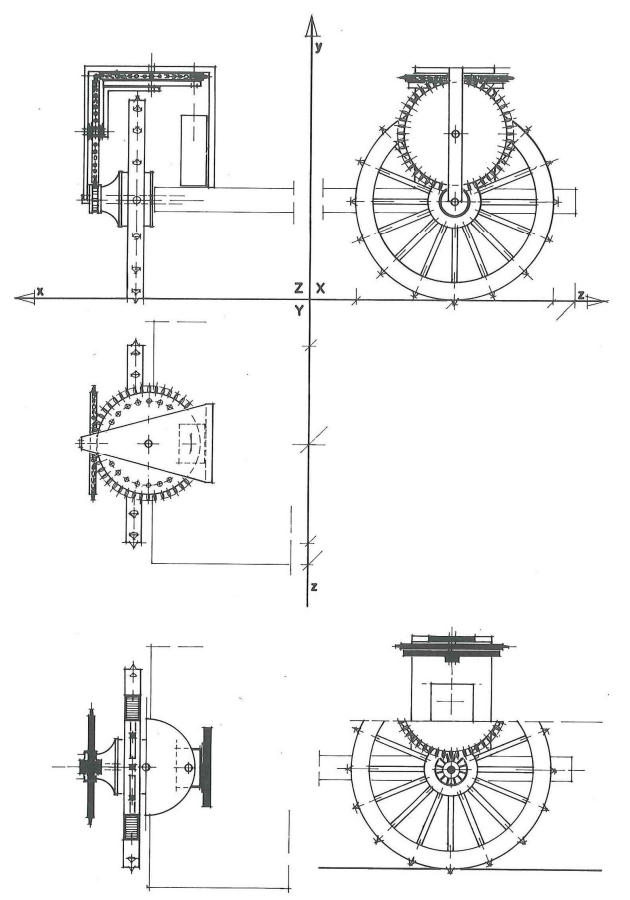

Fig. 1. Ricostruzione dell'odometro di Leonardo (Disegno di M.Carpiceci).

Questi disegni sono composti da una sequenza di segmenti misurati nell'estensione e nell'orientamento. I segmenti costituiscono quindi il percorso seguito da Leonardo per la "poligonazione", ovvero la realizzazione del poligono corrispondente al percorso interno seguito dal Vinci. Che strumenti aveva a disposizione Leonardo?
Uno degli strumenti per la misurazione doveva essere per forza una bussola, probabilmente con la possibilità di effettuare una "traguardazione" e segnarne l'orientamento rispetto ai riferimenti. Ai tempi di Leonardo non esisteva il concetto di un unico riferimento e, quindi, di angolo rispetto al nord magnetico; bensì, vi era la rosa dei venti, utilizzata anche per la navigazione. L'angolo veniva quindi letto rispetto ad un allineamento traguardato ed era riferito ai punti ogni $45^{\circ}$ : Tramontana (nord), Greco (nord-est), Levante, Scirocco, Mezzodì, Libeccio, Ponente e Maestro, con frazioni che davano una ulteriore suddivisione e quindi una maggiore precisione. Nel foglio 12282 retto della raccolta di Windsor Leonardo disegna uno stemma con una bussola, ma non è una bussola semplice (Fig. 1). Vi è rappresentato un meccanismo di rotazione la cui unica funzione può essere quella della collimazione di un punto per dedurne l'orientamento rispetto al punto di osservazione (Carpiceci, 1986, p. 61).

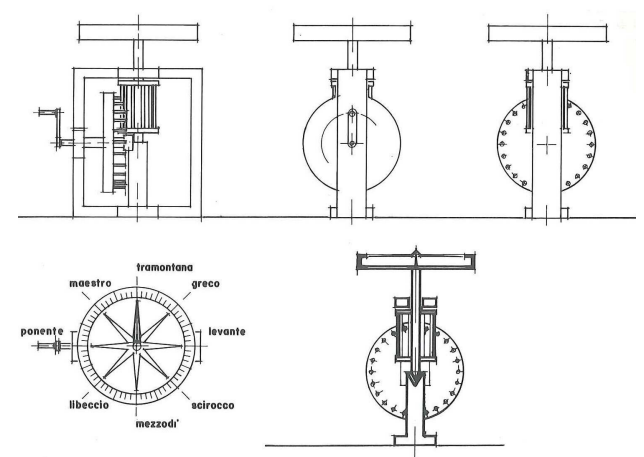

Fig. 2. Ricostruzione della bussola di Leonardo (Disegno di M.Carpiceci).

Leonardo doveva poi possedere uno strumento in grado di misurare la distanza da un vertice al successivo. Ogni volta la distanza era normalmente nell'ordine dei 40-60 m sino a 200 ed oltre. Questo strumento era probabilmente il cosiddetto odometro. Un oggetto utile a questa funzione Leonardo lo disegna nel foglio 1 retto del Codice Atlantico (Fig. 2). Si tratta di un meccanismo comandato da una ruota che ad ogni quantità costante di rotazione fa corrispondere una costante quantità di tragitto percorso dalla ruota. Ogni unità viene "contata" da una 
sfera che cade in un contenitore. Alla fine del percorso la misura è data dalla quantità di sfere nel contenitore (Carpiceci, 1986, p. 55).

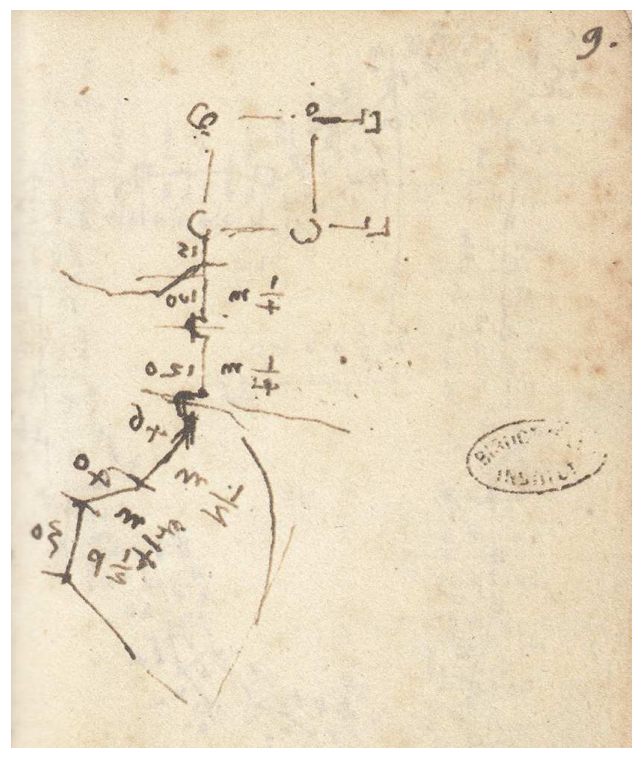

Fig. 3. Leonardo da Vinci, misurazioni delle mura di Cesena. Parigi, Istituto di Francia, Ms L, c.9r.

Successivamente all'operazione di misurazione Leonardo, tornato allo studio, avrebbe dovuto "restituire" le linee in maniera da distribuirle in scala e ricostruire l'intero percorso misurato. Teoricamente, tale operazione applicata ad un circuito chiuso, avrebbe dovuto portare alla chiusura della poligonazione. La prassi, invece, era diversa. L'imprecisione degli strumenti, unita a possibili errori di scrittura, portava ad un inevitabile errore. Quando questo era piccolo, poteva essere ignorato ma quando era grande costringeva il rilevatore, in questo caso Leonardo, ad una ulteriore verifica di alcuni tratti o dell'intero perimetro. Non sono rimasti scritti vinciani relativi a questa operazione come ai disegni di restituzione. Del resto, dal punto di vista puramente teorico, è facile immaginare di percorrere un camminamento di ronda lungo le mura e di aspettarsi prima o poi di ripassare per il punto di partenza. La realtà orografica di un luogo specifico però è spesso tale da disattendere il progetto iniziale. Si possono presentare una miriade di condizioni sottovalutate o ignorate che conducono alla realizzazione di cinte murarie che vengono pragmaticamente chiuse in maniera difforme dall' assunto iniziale.

Grazie al "certosino" lavoro svolto da Nando de Toni (1965) negli anni Sessanta, inspiegabili sequenze di linee e di numeri presenti nel taccuino $\mathrm{L}$ hanno trovato la loro giusta attribuzione geografica ed oggi, con le moderne tecniche di rappresentazione, ne possiamo verificare la precisa collocazione planimetrica. Percorrendo il Codice $\mathrm{L}$ pagina per pagina possiamo riconoscere e interpretare correttamente i vari eidotipi, gli schizzi estemporanei tracciati da Leonardo sul campo durante il rilevamento delle mura di Cesena, Urbino ed Imola. Non è chiaro se la sequenza temporale con la quale sono stati realizzati i disegni coincida con la numerazione delle carte ma decidiamo comunque di seguirla.

\section{Cesena, estate 1502}

Alla carta 9 retto (Fig. 3) vi è un tratto di poligonale che congiunge la porta sud della Città, Porta Montanara, alla Rocca (Fig. 4), schematizzata in un quadrato con quattro torrioni angolari ed altre due torri più ad est.

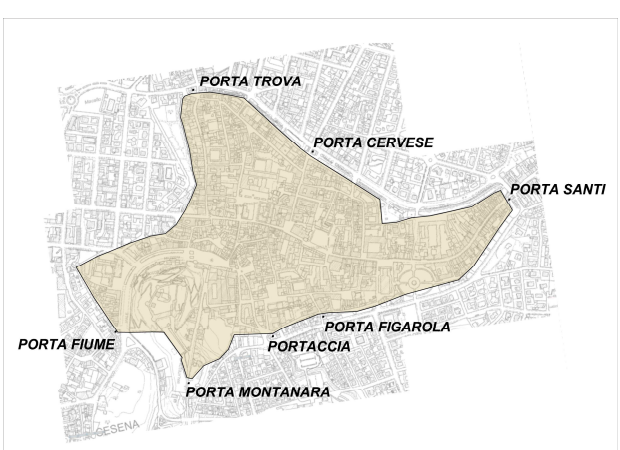

Fig. 4. La cinta muraria di Cesena (Disegno di M.Carpiceci).

Ogni tratto di percorso è segnato con la dimensione, misurata con l'odometro e la direzione del verso del percorso. Leonardo inizia il suo percorso dalla Porta e sale verso la Rocca a nordovest. Quasi tutte le misurazioni indicano come orientamento di riferimento " $\mathrm{m}$ " (maestrale). La bussola utilizzata da Leonardo a Cesena doveva avere la suddivisione classica dei venti: " $\mathrm{t}$ " (Tramontana) nord; "g" (Greco) nord-est; "li" 
(Libeccio) est; "sci" (Scirocco) sud-est; "m" o "1/2di" (Mezzodì) sud; "li" (Libeccio) sudovest; "p" (Ponente); "ma" o "m" o "mo" (Maestrale) nord-ovest. Ogni direzione principale, di ampiezza di $45^{\circ}$, era ulteriormente suddivisa in 8 parti (ogni $5,125^{\circ}$ ) segnate come frazioni, con la sequenza oraria: $1 / 8,1 / 4,3 / 8,1 / 2,5 / 8,3 / 4$ e $7 / 8$.

Quindi ogni orientamento collimato era misurato nella direzione principale con l'aggiunta della frazione misurata in senso orario dall'orientamento stesso. Purtroppo la " $m$ " indica ambiguamente sia il Maestrale che il Mezzodì ma sembra che Leonardo non se ne preoccupi, affidando la successiva decifrazione del dato registrato alla sua memoria e alla logica dell' andamento del percorso.

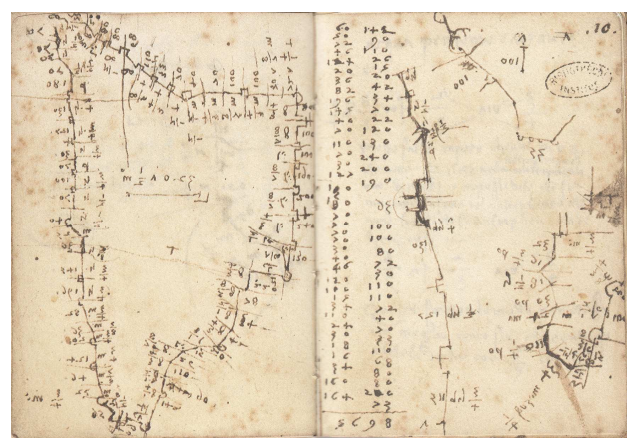

Fig. 5. Leonardo da Vinci, misurazioni delle mura di Cesena. Parigi, Istituto di Francia, Ms L, cc. 9v+10r.

Alla carta 9 verso (Fig. 5, sinistra), prosegue il lavoro di misurazione iniziato nella pagina precedente. Apparentemente Leonardo traccia il perimetro quasi completo delle mura ma l'orientamento rispetto al taccuino è ruotato di $90^{\circ}$. Ci si aspetterebbe che il percorso ricominci dal punto lasciato in precedenza, invece no. Dall'orientamento dei tratti si deduce che il verso, e quindi il movimento, è avvenuto da oriente ad occidente, quindi dalla Porta Santi ad est sino alla Porta Montanara. Nella pagina corrisponde alla spezzata a sinistra che Leonardo quindi deve aver tracciato da sotto a sopra, o da destra a sinistra considerando il taccuino ruotato di $90^{\circ}$ antiorario. Ad ulteriore conferma della stesura 'orientata' degli eidotipi di campagna, vi è una linea tracciata al centro del foglio con vicino la lettera $\mathrm{T}$ a segnalare l'orientamento della Tra- montana, il nord magnetico. Le poligonali tracciate sono, in questo caso, tutte coerentemente orientate. Questo dato, unito al percorso seguito dato dal "verso" degli orientamenti, dimostra che Leonardo, prima di misurare ogni tratto orientato, abbia fatto una sorta di progetto di rilievo: un disegno più o meno corrispondente alla spezzata che avrebbe poi misurato precisamente con l'ausilio degli strumenti. Il tracciato superiore e a destra, corrispondono all' andamento delle mura a nord est e a nord; iniziando dalla torre sul fiume, estrema punta occidentale del recinto, sino alla già citata Porta Santi. Manca quindi una parte del sistema difensivo che collega la Torre occidentale con la porta Montanara a sud. Leonardo non si preoccupa della lacuna planimetrica che verrà colmata nella pagina di fronte, ed inizia il suo camminamento nella sequenza ora descritta sino a ricongiungersi con il varco orientale, Porta Santi. In aggiunta all'eidotipo della complessa poligonale, viene aggiunto un segmento di congiunzione tra la torre occidentale e Porta Fiume a metà strada tra la torre e Porta Montanara.

La pagina di destra del taccuino aperto mostra la carta 10 retto (Fig. 5, destro), dove Leonardo integra ed approfondisce il rilevamento topografico iniziato nelle pagine precedenti. In basso a destra vi è il tratto di poligonale che congiunge la Rocca con la porta a sud-ovest della Città: Porta Fiume. Leonardo lo percorre e misura, concludendo così la fase di misurazione dell'intero perimetro difensivo. Nella stessa pagina Leonardo disegna i tratti di mura intorno a Porta Santi (in alto a destra) e a Portaccia. A sinistra della pagina, trascrive infine su due colonne tutte le misure lineari eseguite per calcolare il perimetro totale: 5698 braccia.

\subsection{La restituzione di Cesena}

A differenza di Leonardo, noi disponiamo di cartografie definite e precise che ci permettono di eseguire dei confronti tra il lavoro svolto nel 1502 e la situazione dei luoghi, che fortunatamente sono rimasti pressoché invariati nei secoli. Dopo aver calcolato, mediante foglio elettronico, tutte le poligonali descritte, relative al perimetro murario di Cesena, le abbiamo ridise- 
gnate con un programma CAD. Abbiamo unito quindi le prime due spezzate, ossia la prima dalla Rocca a Porta Montanara e la seconda da Porta Montanara a Porta Santi. Abbiamo quindi preso la Carta Tecnica Regionale di Cesena (CTR) e, ruotando e ridimensionando la carta stessa, abbiamo iniziato un primo confronto cercando la situazione migliore per la sovrapposizione della poligonale leonardiana all' andamento delle mura ancora esistenti. La sovrapposizione determinata ci è sembrata molto soddisfacente (Fig. 6). Da questa prima operazione si può dedurre che l'inclinazione magnetica del 1502 doveva essere di circa $8,3^{\circ}$ est e che l'unità di misura utilizzata con l'odometro da Leonardo corrispondeva a circa $63 \mathrm{~cm}$, probabilmente pari al braccio.

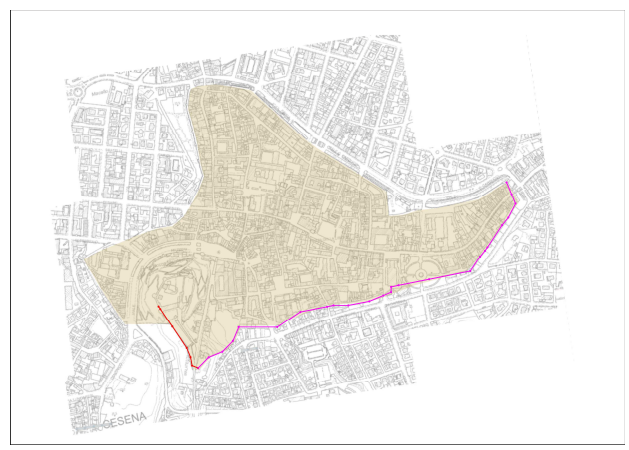

Fig. 6. Restituzione delle prime poligonazioni per Cesena (Disegno di M.Carpiceci).

Abbiamo poi restituito la lunga poligonale che da Porta Santi sale sino al luogo dove era Porta Trova e percorrendo le mura occidentali verso sud arriva sino a Porta Fiume. Da questo punto è stato aggiunto anche il tratto che ricongiunge questa con le mura che scendono dalla Rocca.

Per ragioni di chiarezza "visiva", abbiamo unito le poligonali a partire dalla Rocca e, come prevedibile, la poligonale non si è chiusa (Fig. 7) come sempre avviene in tutte le poligonali topografiche. Nella prassi moderna, data la piccola entità degli errori strumentali, si procede con l'applicazione di una compensazione redistribuendo l'errore lungo la poligonale con intensità calibrate rispetto ad angoli e lunghezze. Nel caso "pionieristico" di Leonardo, questo non può essere fatto, ma merita comunque qualche considerazione.

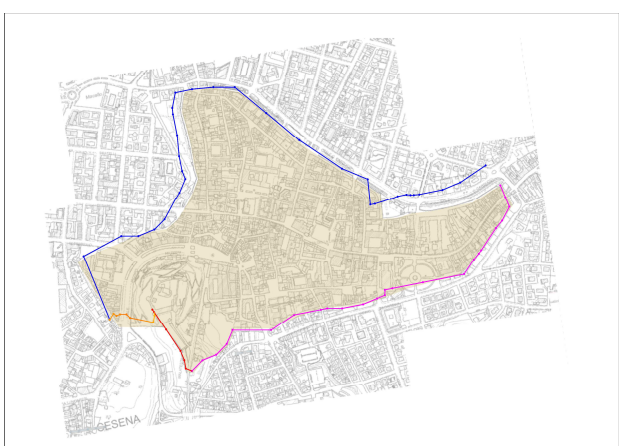

Fig. 7. Restituzione delle poligonazioni per Cesena (Disegno di M.Carpiceci).

Leonardo può aver compiuto errori di trascrizione o di lettura, pertanto qualche misura può essere sbagliata. Inoltre non sappiamo se Leonardo abbia compensato -crediamo di no- l'effetto "ipotenusa" ossia la notevole differenza, nei tratti molto inclinati, tra lunghezza misurata e lunghezza proiettata in orizzontale. La ricerca delle zone di sovrapposizione ha individuato molte zone in cui angoli e distanze sembrano essere "vicini" all'andamento teorico percorso da Leonardo; altre misure sembrano soddisfare le lunghezze ma non l'orientamento, altre sembrano dover essere più corte del misurato, come nella discesa dalla Rocca verso Porta Fiume. In definitiva, limitando un intervento di correzione a soli quattro segmenti del perimetro si è potuta chiudere la poligonale in maniera quasi perfetta (Fig. 8).

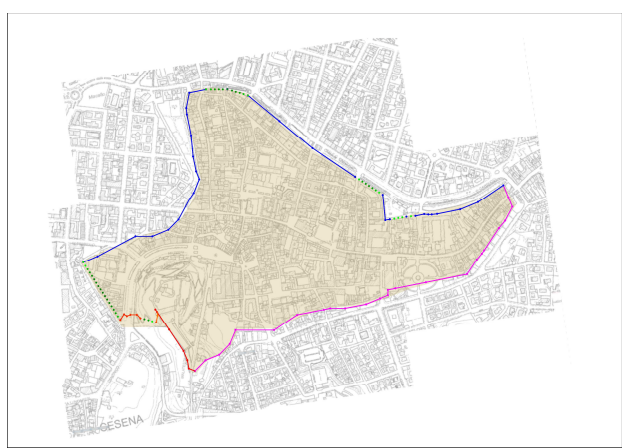

Fig. 8. Restituzione della poligonale chiusa di Cesena con (in verde) le correzioni necessarie (Disegno di M.Carpiceci). 


\section{Urbino, estate 1502}

Mentre la forma delle mura di Cesena è chiara ed evidente nella carta 9 verso, il resto del piccolo taccuino non esplicita alcuna "forma" riconoscibile. Il merito di Nando de Toni (1965) fu di tradurre e restituire i dati numerici in una poligonale confrontabile con le mura delle città che in quel periodo il Vinci avrebbe potuto visitare nelle vesti di "ingegnere delle fortificazioni". Questa prassi lo condusse a confrontare la poligonale con il perimetro delle mura di Urbino (Fig. 9) la cui figura, con somma sorpresa, era nascosta in due diversi punti del Taccuino: le due pagine raffrontate nelle carte 37 verso e 38 retto e quelle nelle carte 74 verso e 75 retto.

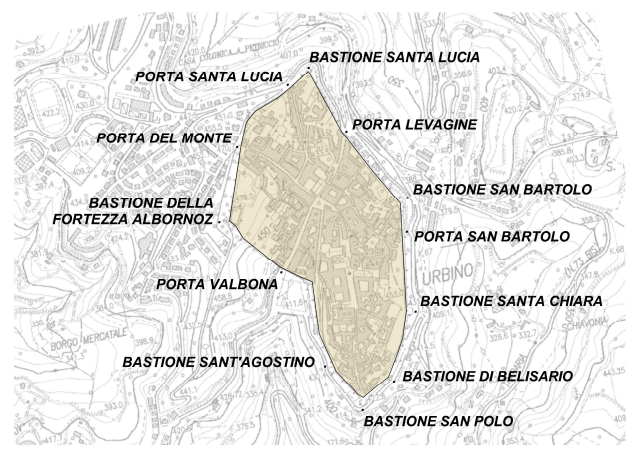

Fig. 9. La cinta muraria di Urbino (Disegno di M.Carpiceci).

Differentemente da Cesena, le due coppie di pagine presentano una forma di notazione comune. Da una parte c'è un disegno non orientato del percorso seguito con la misura delle sole lunghezze. Nella pagina a fronte vi sono solo i dati numerici con la ripetizione delle lunghezze e l'aggiunta degli orientamenti. Ci sono però delle differenze per quanto riguarda l'orientamento, che sembrano confermare l'ipotesi di de Toni che Leonardo abbia utilizzato qui una bussola differente. Mentre la suddivisione angolare dei $45^{\circ}$ adottata per Cesena è in due, quattro e otto parti, qui si aggiunge inspiegabilmente la terza parte. Questa integrazione però non incrementa omogeneamente la suddivisione, come avrebbe potuto fare una suddivisione in 16 , bensì aggiunge i soli due orientamenti di $1 / 3$ e $2 / 3$ che risultano intermedi tra $1 / 4$ e $3 / 8$ e tra $1 / 2$ e $5 / 8$, producendo una disomogenea distribuzione delle misure angolari. Forse intento a sperimentare varianti operative, Leonardo segna due orientamenti: il primo è l'orientamento principale ed il secondo quello verso il quale è presa la misura frazionaria. Ne consegue che il dato della frazione può essere orario o antiorario a seconda della direzione secondaria specificata. Questa decisione ha quindi come risultato l'aggiunta apparentemente non necessaria- di un ulteriore dato e quindi di un ulteriore possibile errore di trascrizione. La ripetizione delle misure lineari certamente è pensata per rendere più pulita la grafica degli eidotipi, ma anche questo passaggio comporta un ulteriore rischio di possibili errori di trascrizione. Infatti, non sempre i numeri sono coincidenti. D'altro canto, questa doppia lista potrebbe anche indicare una ripetizione delle misurazioni.

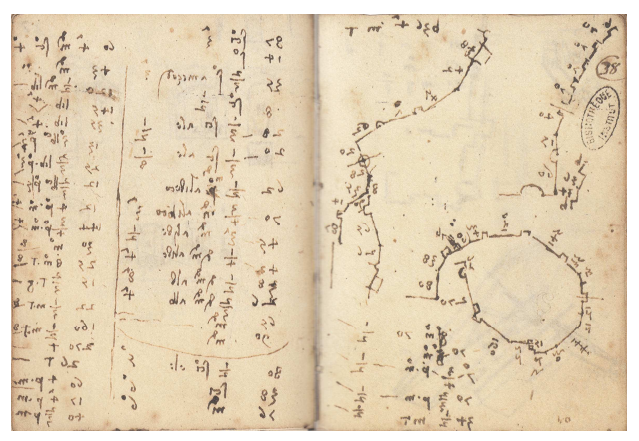

Fig. 10. Leonardo da Vinci, misurazioni delle mura di Urbino. Parigi, Istituto di Francia, Ms L, cc. $37 v+38 r$.

Nella coppia di fogli $37 \mathrm{v}-38 \mathrm{r}$ (Fig. 10) viene analizzato il tratto meridionale delle mura di Urbino. In base al verso degli orientamenti si è individuata un'unica poligonale che percorre il perimetro meridionale da un punto, non meglio identificato, a nord-ovest del bastione San Bartolo, sino al bastione San Polo (estrema punta meridionale), continuando poi sino alla Porta Valbona ad est.

La coppia di fogli 74v-75r (Fig. 11) è più complessa. Leonardo concentra in queste due pagine tutto il rilievo delle mura ripercorrendo anche la zona meridionale. In aggiunta, inizia anche la collocazione di alcune strade principali della città che collegano il centro con le porte Levagine, del Monte e Valbona. L'operazione di "poligo- 
nazione" non è unica come il caso precedente ma si divide in più settori. C'è però una costante di orientamento, ovvero i percorsi procedono sempre da sud verso nord, e questo sia per le misurazioni lungo le mura, sia per i tre percorsi interni che congiungono Porta Valbona con la piazza centrale e che poi si dividono verso Porta Levagine a nord-est o verso Porta del Monte a nord-ovest. Alla carta 75r il numero 3827 ci mostra che anche in questo caso il risultato del calcolo del perimetro totale in braccia.

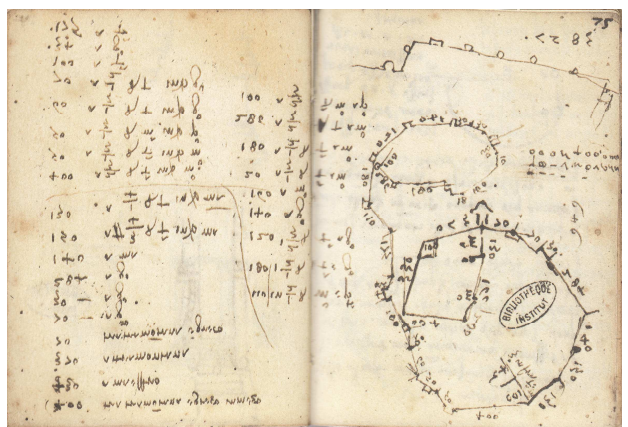

Fig. 11. Leonardo da Vinci, misurazioni delle mura di Urbino. Parigi, Istituto di Francia, Ms L, cc. 74v+75r.

\subsection{La restituzione di Urbino}

La variabilità degli appunti metrici e degli eidotipi ha reso la restituzione non sempre facile, visto che anche l'orientamento del foglio, e quindi delle scritte, subisce continue rotazioni e ribaltamenti, in aggiunta alla stesura non sempre sinistrorsa. In analogia con Cesena abbiamo calcolato l'estensione dei tratti rettilinei considerando il braccio equivalente a $63 \mathrm{~cm}$. Nel cercare di sovrapporre le due poligonali tra di loro e sulla planimetria, si sono evidenziati numerosi errori, sia di lunghezza che di orientamento (Fig. 12).

Va notato che il tratto ad est tra i bastioni San Bartolo e Santa Chiara, che attualmente realizzano un tratto rettilineo delle mura, nelle misure di Leonardo segue l'andamento delle case più interne. Questo suggerisce che quel tratto o era stato distrutto per eventi bellici o ancora seguiva un andamento più mistilineo, come pure il tratto più a settentrione tra Porta Levagine e il bastione Santa Lucia.

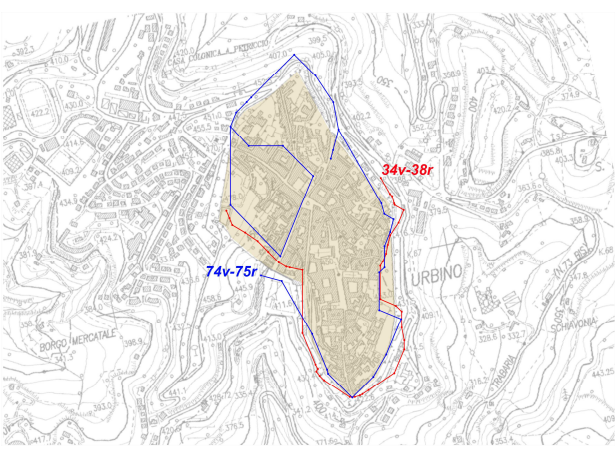

Fig. 12. Restituzione delle poligonazioni per Urbino (Disegno di M.Carpiceci).

La sovrapposizione del semiperimetro meridionale ha permesso di capire quale dei due sistemi poligonali andava scelto. Questo ha portato ad una miscela "controllata" dei due sistemi alla quale integrata delle necessarie correzioni. Il risultato chiaramente è una nostra ricostruzione a posteriori (Fig. 13), che permette di evidenziare i più grossolani errori ma anche le varianti misurate rispetto all'attuale stato dei luoghi.

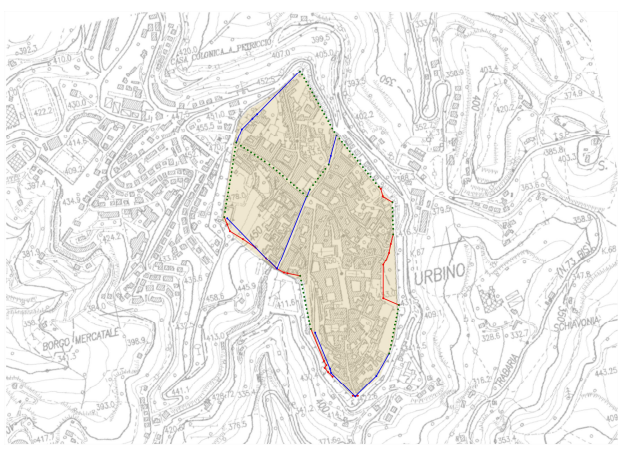

Fig. 13. Restituzione della poligonale chiusa di Urbino con (in verde) le correzioni necessarie (Disegno di M.Carpiceci).

\section{Conclusioni}

L'analisi di alcune pagine del Taccuino $\mathrm{L}$ ha messo in luce il modus operandi adottato da Leonardo da Vinci nel rilievo delle mura di Cesena ed Urbino. È probabile che tale metodo, che prevede l'effettiva percorribilità del perimetro delle mura, non potesse essere utilizzato sempre o che in altri casi, come gli insediamenti che potevano essere dominati da un punto elevato, egli preferisse ricorrere a procedure ottiche, proietti- 
ve o trigonometriche. Ad ogni modo, quelle analizzate in questo paper, sono le uniche rilevazioni di cinte murarie eseguite per poligonazione da Leonardo da Vinci. Sembra possibile che Leonardo non abbia più portato avanti l'elaborazione di questa tecnica per mancanza di sufficienti strumenti analitici per eseguire la compensazione delle misure. Certamente, dal punto di vista "statistico", avrebbe potuto reiterare il processo con gli stessi punti di stazione. In questa maniera avrebbe potuto trovare $\mathrm{i}$ dati ricorsivi che gli avrebbero mostrato dimensioni e orientamenti simili e quindi più "probabilmente" giusti; ed avrebbe potuto così richiudere le poligonali perimetrali e le eventuali misure interne della principale viabilità cittadina.
Nel codice L l'unico ulteriore disegno urbano è quello relativo al porto di Cesenatico, ma non affronta in alcun modo i suoi eventuali apparati difensivi (Beltrami 1902). Al di fuori del piccolo taccuino del 1502, esiste un altro rilevamento urbano con relative mura ed è quello della città di Imola. Al foglio 12686r. a Windsor, Leonardo ne disegna la rete viaria annotando anche le lunghezze dei tratti. Al foglio 12284, sempre a Windsor, c'è la celeberrima pianta. Nonostante l'intervento diretto di Leonardo su questa mappa non possa essere messo in discussione, diversi studiosi (Mancini, 1979; Toni, 1982; Friedman, 2010) hanno messo in luce il suo debito "cartografico" nei confronti dell'ingegnere Danesio Maineri, su cui ci ripromettiamo di investigare in una prossima occasione.

\section{Bibliography}

Beltrami, L. (1902). Leonardo e il porto di Cesenatico, Milano.

Carpiceci, M. (1986). Leonardo, la misura e il segno, Roma.

Friedman, D. (2010). "La pianta di Imola di Leonardo, 1502", in Rappresentare la città: topografie urbane nell'Italia di antico regime, Reggio Emilia, pp. 121-144.

Mancini, F. (1979). Urbanistica rinascimentale a Imola da Giacomo Riario a Leonardo da Vinci (1474-1502), Imola.

Marani, P. (1984). L'architettura fortificata negli studi di Leonardo da Vinci, Firenze.

Toni, N. de. (1957). "Leonardo da Vinci e i rilievi topografici di Cesena", Studi Romagnoli, 8, pp. 414-424.

Toni, N. de. (1965). "I rilievi cartografici di Leonardo per Cesena ed Urbino contenuti nel manoscritto 'L' dell'Istituto di Francia", in V Lettura Vinciana, Firenze.

Toni, N. de. (1982). "La pianta d'Imola e Leonardo", Raccolta Vinciana, 21, pp. 21-32. 